\title{
Excitation of zero-frequency magnetic field-aligned currents by ionospheric heating
}

\section{A. V. Streltsov and T. R. Pedersen}

Thayer School of Engineering, Dartmouth College, Hanover, NH 03755, USA

Space Vehicles Directorate, Air Force Research Laboratory, Hanscom AFB, MA 01731, USA

Received: 24 January 2011 - Revised: 12 May 2011 - Accepted: 7 June 2011 - Published: 27 June 2011

\begin{abstract}
Time-dependent, three-dimensional numerical simulations of the reduced MHD model describing shear Alfvén waves in the magnetosphere provide an interesting prediction superficially similar to results of several ionospheric heating experiments conducted at high altitudes. In these experiments, heating of the ionospheric F-region with a constant/zero-frequency beam of HF waves causes luminous structures in the ionosphere in the form of a ring or a solid spot with a characteristic size comparable to the size of the heated spot. Simulations suggest that spots/rings or similar optical appearance might be associated with a magnetic fieldaligned current system produced by the ionospheric heating. Two of the most interesting features of this current system are (1) strong localization across the ambient magnetic field and (2) distinctive non-symmetrical luminous signatures (ring/spot) in magnetically conjugate locations in the ionosphere.
\end{abstract}

Keywords. Ionosphere (Active experiments; Ionospheremagnetosphere interactions) - Magnetospheric physics (MHD waves and instabilities)

\section{Introduction}

Heating of the ionosphere with powerful HF transmitters can cause many different physical phenomena occurring at the same time at the same location. These phenomena include "mostly ionospheric" processes, like generation of the ionospheric plasma instabilities and irregularities, and waves propagating in the ionosphere and in the ionosphereatmosphere wave guide. They also include "mostly magnetospheric" processes, like generation of ULF and ELF

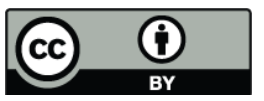

Correspondence to: A. V. Streltsov (streltsov@dartmouth.edu) waves propagating into the magnetosphere, interactions between these waves and particles in the magnetosphere, particle precipitation into the ionosphere, and secondary ionospheric phenomena caused by this precipitation (e.g., auroral arcs). Due to the strong electromagnetic coupling between the ionosphere and the magnetosphere, particularly in the ULF frequency range, both magnetospheric and ionospheric geophysical processes may contribute to the results of the heating experiments observed with ground magnetometers, ULF receivers, and optical instruments. In most cases, a specific mechanism cannot be conclusively identified from the experimental data, and effects with multiple potential sources are particularly problematic.

An example of an effect with potentially multiple source mechanisms is luminous structures in the form of rings or solid spots registered with all-sky cameras during several heating experiments conducted at the High Frequency Active Auroral Research Program (HAARP) facility in Alaska. Artificial airglow excited by ionospheric heating with powerful HF waves has been reported in numerous experiments, e.g. Bernhardt et al. (1988, 1989). In this paper, we focus on events, like the one shown in Fig. 1, where the image from a $630.0 \mathrm{~nm}$ camera taken $\approx 1$ min into a 12 -min long, zero-frequency heating period (03:00:00-03:11:50 UT) on 11 November 2009 is shown. Heating was performed with 3.6 MW, O-mode, $2.85 \mathrm{MHz}$ waves pointed into the magnetic zenith. Similar observations of ring-like structures have been presented and discussed in detail by Pedersen et al. (2009), who suggested that these structures are produced by refraction from localized density enhancements in the ionosphere caused by the heating.

We suggest that in addition to that effect, density in the ionosphere can also be enhanced locally by precipitation of the magnetospheric electrons caused by ULF waves standing along the magnetic field lines between the magnetically conjugate locations in the magnetosphere, inside a so-called global magnetospheric resonator. Shear Alfvén waves inside

Published by Copernicus Publications on behalf of the European Geosciences Union. 
this resonator have been studied for more than 50 years with a goal of explaining geomagnetic pulsations in the Pc5 frequency range frequently observed at high latitudes, particularly during substorms (Cummings et al., 1969; Southwood, 1974; Ruohoniemi et al., 1991; Samson et al., 1992). Because these waves carry magnetic field aligned currents (FACs) which, under some conditions, can develop parallel electric field and cause electron precipitation into the ionosphere, they have also been extensively studied in connection with bright, discrete auroral arcs (Xu et al., 1993; Streltsov et al., 1998; Streltsov, 1999; Stasiewicz et al., 2000). Various physical mechanisms causing precipitation of magnetospheric electrons into the ionosphere by ULF waves (like wave dispersion, anomalous resistivity, temperature anisotropy and the mirror force) also have been studied quite extensively, and this subject is not considered in this paper. We only want to emphasize that all of these mechanisms relate the parallel electric field (and the parallel electron acceleration) to the FAC in the wave, and therefore, we limit our study to the investigation of how the ionospheric heating with zero-frequency modulation of an HF beam can generate large-amplitude Alfvén waves/FACs in the magnetosphere.

\section{Model}

Propagation of shear Alfvén waves along magnetic field lines at HAARP latitudes (near $L=4.9$ dipole magnetic shell) can be described with a reduced, two-fluid MHD (RMHD) model (Streltsov et al., 1998). The linearized model includes the parallel component of the electron momentum equation, the density continuity equation, and the current continuity equation:

$$
\begin{aligned}
& \frac{\partial v_{\| e}}{\partial t}+\frac{e}{m_{\mathrm{e}}} E_{\|}+\frac{1}{m_{\mathrm{e}} n_{0}} \nabla_{\|}\left(n T_{\mathrm{e}}\right)=-v_{\mathrm{e}} v_{\| e}, \\
& \frac{\partial n}{\partial t}+\nabla \cdot\left(n v_{\| \mathrm{e}} \hat{\mathbf{b}}\right)=0, \\
& \left(1-\rho_{i}^{2} \nabla_{\perp}^{2}\right) \nabla \cdot j_{\|} \hat{\mathbf{b}}+\frac{1}{\mu_{0}} \nabla \cdot\left(\frac{1}{c^{2}}+\frac{1}{v_{\mathrm{A}}^{2}}\right) \frac{\partial \mathbf{E}_{\perp}}{\partial t}=0 .
\end{aligned}
$$

Here, the subscripts $\|$ and $\perp$ denote vector components in the directions parallel and perpendicular to $\hat{\mathbf{b}}=\mathbf{B}_{0} / B_{0} ; v_{\| \mathrm{e}}$ is the parallel component of the electron velocity; $v_{\mathrm{A}}=$ $B_{0} / \sqrt{\mu_{0} n_{0} m_{\mathrm{i}}}$ is the Alfvén speed; $\rho_{\mathrm{i}}=T_{\mathrm{i}}^{1 / 2} / m_{\mathrm{i}}^{2} \omega_{c i}$ is the ion Larmour radius; $T_{\mathrm{e}, \mathrm{i}}$ is the background electron/ion temperature; and $\nu_{\mathrm{e}}$ is the electron collision frequency. The electric field and the magnetic field-aligned current are expressed in terms of the scalar potential, $\phi$, and the parallel component of the vector potential, $A_{\|}$, as $\mathbf{E}_{\perp}=-\nabla_{\perp} \phi$, $E_{\|}=-\nabla_{\|} \phi-\partial A_{\|} / \partial t, j_{\|}=-e n v_{\| \mathrm{e}}=-\nabla_{\perp}^{2} A_{\|} / \mu_{0}$.

The 3-D numerical algorithm and the computational grid used to solve Eqs. (1)-(4) are natural extensions of the previously used and well-documented 2-D algorithm for the same RMHD model (Streltsov et al., 1998). It is based on a "finite-difference-time-domain" (FDTD) numerical technique in a three-dimensional computational domain bounded by $L=4.75$ and $L=5.05$ magnetic shells and two azimuthal planes separated by $1.91^{\circ}$ in longitude. These boundaries define a banana-like domain in the magnetosphere which has a square "footprint" in the ionosphere (100 km altitude), with the length of the square side of $\approx 98 \mathrm{~km}$. The values of $\phi$ and $A_{\|}$are set equal to 0 on $L=4.75$ boundary of the domain, and the normal derivatives of $\phi$ and $A_{\|}$are set equal to 0 on $L=5.05$ boundary. Periodic boundary conditions are implemented on the longitudinal surfaces of the domain. The code employs a grid which is non-uniform along the ambient magnetic field, with the parallel cell size decreasing 50 times from the equator to the ionosphere. Thus by having only 101 nodes along the entire $L=4.9$ magnetic field line this grid provides spatial resolution of $\approx 47.5 \mathrm{~km}$ near the ionosphere. The ionospheric boundaries of the domain are set at $100 \mathrm{~km}$ altitude. There, the scalar potential, $\phi$, defining the perpendicular electric field is connected with the parallel current density, $j_{\|}$, via the Poisson equation

$$
\nabla \cdot\left(\Sigma_{\mathrm{P}} \nabla_{\perp} \phi\right)= \pm j_{\|}
$$

Here, $\Sigma_{\mathrm{P}}=M_{\mathrm{P}} n_{\mathrm{E}} h e / \cos \alpha$ is the height-integrated Pedersen conductivity; $M_{\mathrm{P}}=10^{4} \mathrm{~m}^{2} \mathrm{sV}^{-1}$ is the ion Pedersen mobility; $n_{\mathrm{E}}$ is the height-averaged E-layer plasma density; $h=20 \mathrm{~km}$ is the effective thickness of the ionospheric Elayer; $e$ is the elementary charge; $\alpha=14.4^{\circ}$ is the angle between the normal to the ionosphere and the $L=4.9$ dipole magnetic field line at $100 \mathrm{~km}$ altitude. The sign "--" appears in the northern boundary condition and the sign "+" appears in the southern boundary condition.

Relation (4) assumes that the ionospheric density/conductivity is homogeneous (in this case the Hall conductivity is not included in Eq. 4) and constant in time. These assumptions work well for modeling heating experiments when the background conductivity is relatively high ( $\geq 5-10 \mathrm{mho}$ ), and the amplitude of the waves/FACs is relatively small $\left(j_{\|} \leq 1 \mu \mathrm{A} \mathrm{m}^{-2}\right)$. When the background density is low and/or the amplitude of the waves reaches significant amplitudes the Hall conductivity should be taken into the consideration and the ionospheric boundary condition should incorporate effects of active magnetosphere-ionosphere interactions (the so-called ionospheric feedback mechanism), e.g., Streltsov and Pedersen (2010).

\section{Background parameters}

The ambient magnetic field is assumed to be a dipole: $B_{0}=$ $B_{*}\left(1+3 \sin ^{2} \theta\right)^{1 / 2} / r^{3}$, where $B_{*}=31000 \mathrm{nT} ; \theta$ is a colatitudinal angle; and $r$ is a geocentric distance measured in $R_{\mathrm{E}}=6371.2 \mathrm{~km}$.

The background density is modeled as

$$
n(r)= \begin{cases}a_{1}\left(r-r_{2}\right)+a_{2}, & r_{1}<r<r_{2} \\ b_{1} e^{-\left(r-r_{2}\right) / r_{0}}+b_{2} / r, & r>r_{2}\end{cases}
$$




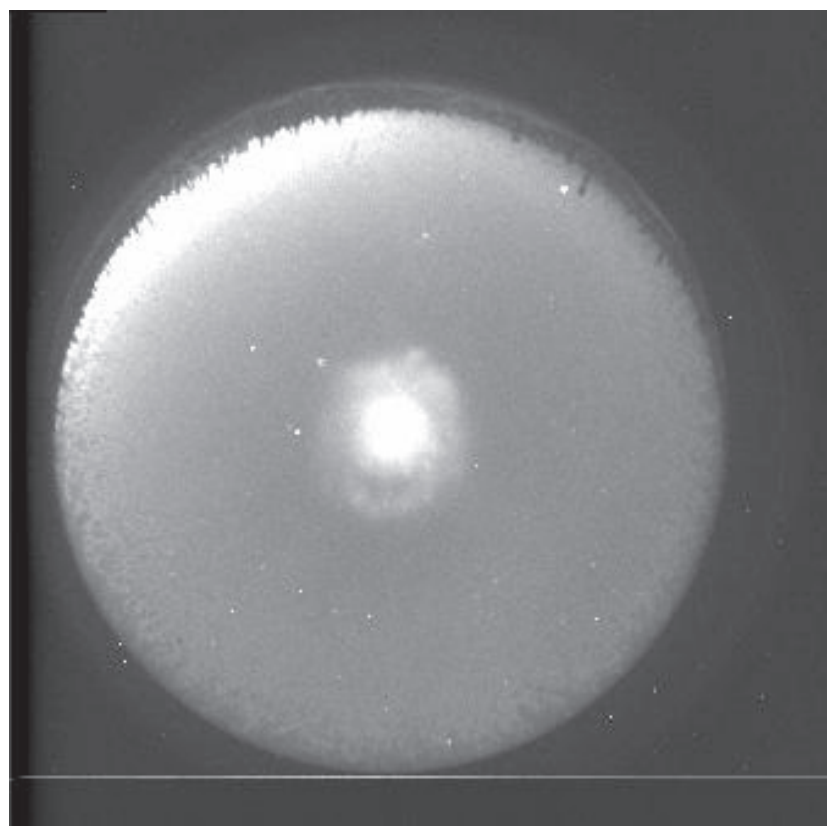

Fig. 1. Image of the luminous structure observed on 11 November 2009 at HAARP with the $630.0 \mathrm{~nm}$ all-sky camera during heating of the ionospheric F-layer with 3.6 MW, O-mode, 2.85 MHz waves pointed into the magnetic zenith.

Here, $r_{0}=0.0188 ; \quad r_{1}=1+100 / R_{\mathrm{E}} ; r_{2}=1+220 / R_{\mathrm{E}}$; and the constants $a_{1}, a_{2}, b_{1}$, and $b_{2}$ are chosen to provide a density of $1.4 \times 10^{5} \mathrm{~cm}^{-3}$ at the altitude $100 \mathrm{~km}$ (Eregion); $2.5 \times 10^{5} \mathrm{~cm}^{-3}$ at the altitude $220 \mathrm{~km}$ (F-region); and $129 \mathrm{~cm}^{-3}$ at the equatorial magnetosphere at the geocentric distance of $4.9 R_{\mathrm{E}}$. These density values are similar to the values used by Streltsov et al. (2010), where they provide good, quantitative agreement between the model results and the observations of VLF waves propagation along the $L=4.9$ magnetic field line. For the parameters of the ionosphere discussed in the previous section, a density value of $1.4 \times 10^{5} \mathrm{~cm}^{-3}$ provides $\Sigma_{\mathrm{P}}=4.5 \mathrm{mho}$ in the E-region.

Electron and ion temperatures are defined as $T_{\mathrm{e}, \mathrm{i}}=$ $T_{\mathrm{e}, \mathrm{i}}^{*}\left(n^{*} / n\right)$, where the constants $T_{\mathrm{e}}^{*}=2 \mathrm{eV}, T_{\mathrm{i}}^{*}=2 \mathrm{eV}$, and $n^{*}=129 \mathrm{~cm}^{-3}$ represent corresponding quantities in the equatorial plane on the $L=4.9$ magnetic shell. The relation between $n_{0}$ and $T_{\mathrm{e}, \mathrm{i}}$ nullifies the parallel gradient in plasma pressure and provides an equilibrium state in the model.

\section{Results and discussion}

ULF waves in the magnetosphere can be generated by ionospheric heating via several different physical mechanisms. The most common one is that the heating changes plasma density/conductivity in the E-region, and modulations in conductivity lead to modulations in the ionospheric currents driven by the large-scale electric field, normally associated with the electrojet. According to Eq. (4), oscillations of
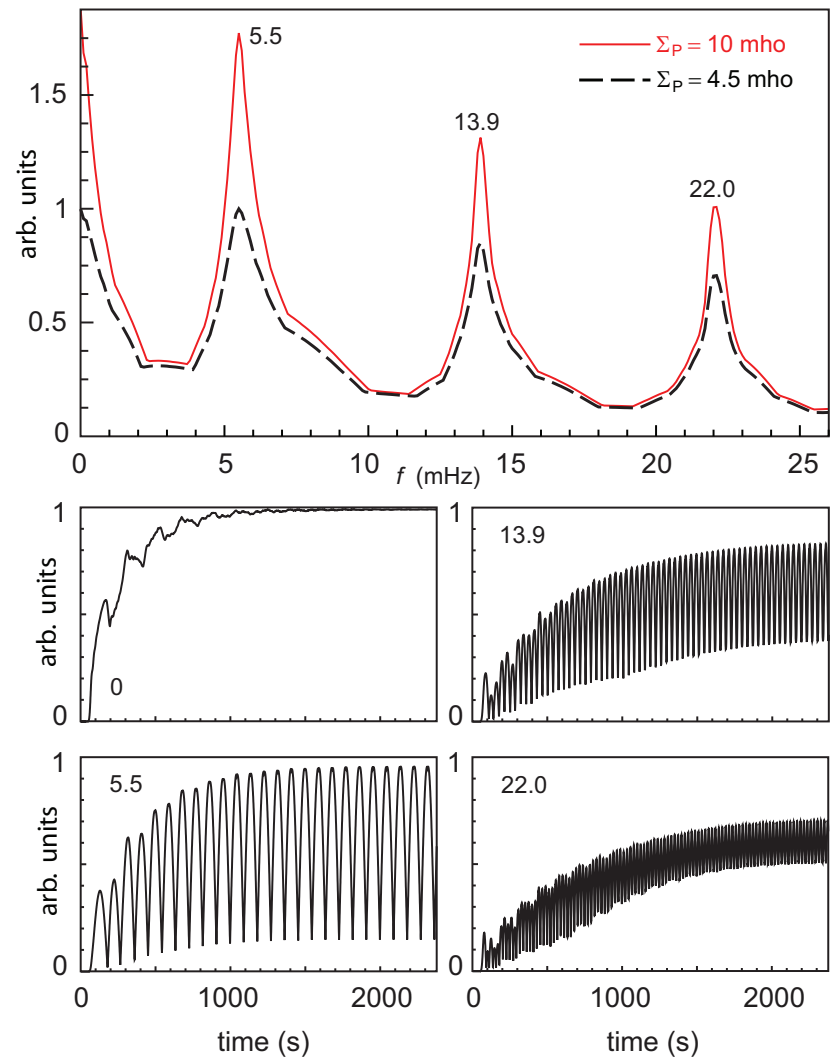

Fig. 2. Top: maximum amplitude of $B_{\perp}$ in the ionosphere obtained during $2377 \mathrm{~s}$ of 2-D simulations with $\Sigma_{\mathrm{P}}=10 \mathrm{mho}$ (red, solid line) and $\Sigma_{\mathrm{P}}=4.5$ mho (black, dashed line). Bottom: dynamics of $\left|B_{\perp}\right|$ in the southern ionosphere in 2-D simulations with $\Sigma_{\mathrm{P}}=4.5$ mho and driver's frequencies of $0,5.5,13.9$, and $22.0 \mathrm{mHz}$.

the ionospheric current are coupled with oscillations of the magnetic field-aligned currents (FACs), which are carried by shear Alfvén waves in the magnetosphere (Yampolski et al., 1997; Robinson et al., 2000; Papadopoulos et al., 1990; Cohen et al., 2010). A recent study by Papadopoulos et al. (2011) demonstrates that shear Alfvén waves can also be generated by variations in the plasma pressure in the ionospheric F-region caused by the heating. The time-varying pressure generates fast MHD waves, which carry perpendicular electric field (or scalar potential) to the E-region, where this field couples to the magnetic filed-aligned currents (or shear Alfvén waves). Therefore, this pressure driver can generate ULF waves even without a background electric field, which makes it very important at low latitudes and or at high latitudes when there is no the electrojet.

Because Eqs. (1)-(3) describe only magnetically incompressible shear Alfvén waves, the ionospheric physics responsible for the wave generation with the pressure driver is not included in the model. This is an obvious shortcoming of the model which we intend to address in feature studies. In this study the waves are excited by the driver in the northern ionospheric boundary described with an additional 

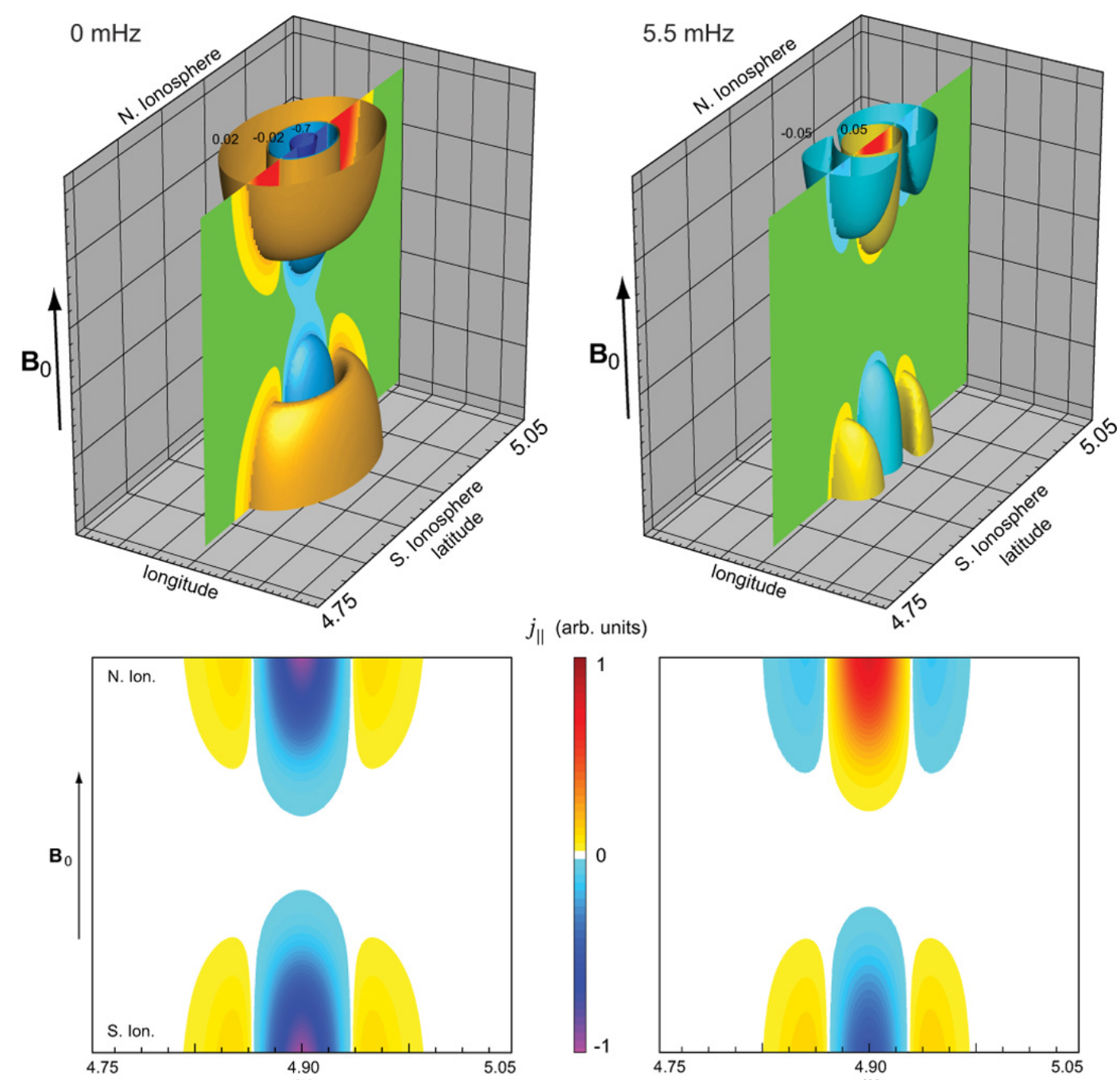

Fig. 3. Left, top: parallel current density, $j_{\|}$, in the magnetosphere obtained in the 3-D simulation with zero-frequency driver at time $t=900 \mathrm{~s}$; left, bottom: $j_{\|}$in the meridional plane cut through the center of the current structure. Right: the same quantities obtained in the 3-D simulation with $5.5 \mathrm{mHz}$ driver.

term to the scalar potential $\phi$ in the boundary condition (4). This driver is proportional to $e^{-x^{2}} \sin (2 \pi f t)$, where $f$ is the frequency of the modulation of HF waves an when $f=0$ the driver term is proportional to $e^{-x^{2}} ; x=\rho / \rho_{0} ; \rho$ is the distance from the center of the ionospheric "footprint" of the computational domain and $\rho_{0}=10 \mathrm{~km}$ is the scale factor, which is chosen to make the width of the heating spot $\sim 20 \mathrm{~km}$ at $100 \mathrm{~km}$ altitude. This is the minimal size of the heating spot achieved by the HAARP in the E-region. The size of the heating spot in the F-region is expected to be larger by a factor of 2 or 3 due to the divergence of the beam.

To excite standing Alfvén waves eigenfrequencies of the magnetospheric field line resonance at $L=4.9$ magnetic shell should be determined. One way to do that is to solve one-dimensional eigenvalue equations derived for these waves in the dipole magnetic field geometry by Cummings et al. (1969) or Streltsov (1999). We find the eigenfrequencies by solving in time a two-dimensional, axisymmetrical (all azimuthal derivatives are set equal to 0) set of Eqs. (1)(4) by changing the frequency of the ionospheric driver from 0 to $26 \mathrm{mHz}$ with an increment of $0.1 \mathrm{mHz}$. The maximum amplitude of the perpendicular magnetic field, $B_{\perp}$, observed during the simulation time of $2377 \mathrm{~s}$ with ionospheric conductivity $\Sigma_{\mathrm{P}}=10 \mathrm{mho}$ (red, solid line) and $\Sigma_{\mathrm{P}}=4.5 \mathrm{mho}$ (black, dashed line) is shown in the top panel in Fig. 2. (Because this is a linear model, we assign value 1 to the saturated amplitude of the magnetic field produced by a zerofrequency driver when $\Sigma_{\mathrm{P}}=4.5 \mathrm{mho}$.) Figure 2 shows that there are three very distinctive resonances with eigenfrequencies of 5.5, 13.9, and $22.0 \mathrm{mHz}$. It also shows that the amplitude of $B_{\perp}$ caused by a constant heating of the ionosphere is comparable with or even greater than the amplitude of $B_{\perp}$ 
caused by the heating with other, non-zero eigenfrequencies of the system. This effect is further illustrated in the lower panels in Fig. 2, where the maximum amplitudes of $B_{\perp}$ in the ionosphere are shown as a function of time in simulations driven with frequencies of $0,5.5,13.9$, and $22.0 \mathrm{mHz}$.

To study the structure of FACs produced by the constant heating, simulations of Eqs. (1)-(4) were performed in a dipole 3-D geometry. The top panels in Fig. 3 show the structure of $j_{\|}$obtained in 3-D simulations with a zero-frequency driver (on the left) and $5.5 \mathrm{mHz}$ driver (on the right) at time $t=900 \mathrm{~s}$. The bottom panels in Fig. 3 show the meridional (constant longitude) cut through the three-dimensional $j_{\|}$shown in the top panels. (Again, because this is a linear model, we assign value 1 to the saturated amplitude of $j_{\|}$ produced by a zero-frequency driver when $\Sigma_{\mathrm{P}}=4.5 \mathrm{mho}$ and use the same color pallet in both 2-D plots shown in Fig. 3 . The positive current flows in the direction of the ambient magnetic field.) The first conclusion from these simulations is that a zero-frequency heating leads to a rapid formation of an axisymmetrical, localized across the ambient magnetic field and extended along the entire dipole magnetic flux tube, "pipe-like" FAC system. In this structure, the field-aligned current flows from one hemisphere to another in the center of the pipe and return back along the outer side of the pipe. In this case, if these FACs cause any parallel electric field in the magnetosphere (through the mechanisms not considered in this paper) which will energize and precipitate electrons into the ionosphere, then an observer at HAARP should see a discrete aurora in the form of a bright spot, whereas an observer in the magnetically conjugate location should see a luminous ring (or vice versa, depending on the sign of the current driver).

Zero-frequency rings can be observed in both hemispheres if the heater power is distributed across the magnetic field not like $e^{-x^{2}}$ but rather like $e^{-x^{6}}$. In other words, luminous rings will be produced in both hemispheres if the heated spot has very sharp boundaries. This heated spot will create the "pipelike" current system where the current in one direction flows along the internal surface of the pipe and the return current flows along the external surface of the pipe. This situation is illustrated in the right panels in Fig. 4. The color pallet in this figure has the range from -1.0 to 0.5 , where value 1 corresponds to the saturated amplitude of $j_{\|}$produced by a zero-frequency driver when $\Sigma_{\mathrm{P}}=4.5 \mathrm{mho}$.

In conclusion, 3-D simulations of shear Alfvén waves presented in this paper indicate that heating of the ionosphere with a constant/zero-frequency beam of HF waves could potentially cause luminous structures in the form of a ring or a solid spot with a characteristic size comparable with the size of the heated spot. These spots/rings are produced by electrons precipitating from the magnetosphere by the parallel electric field/parallel potential drop associated with a FAC system produced by the heating. Two of the most interesting features of the Alfvén wave-related current system
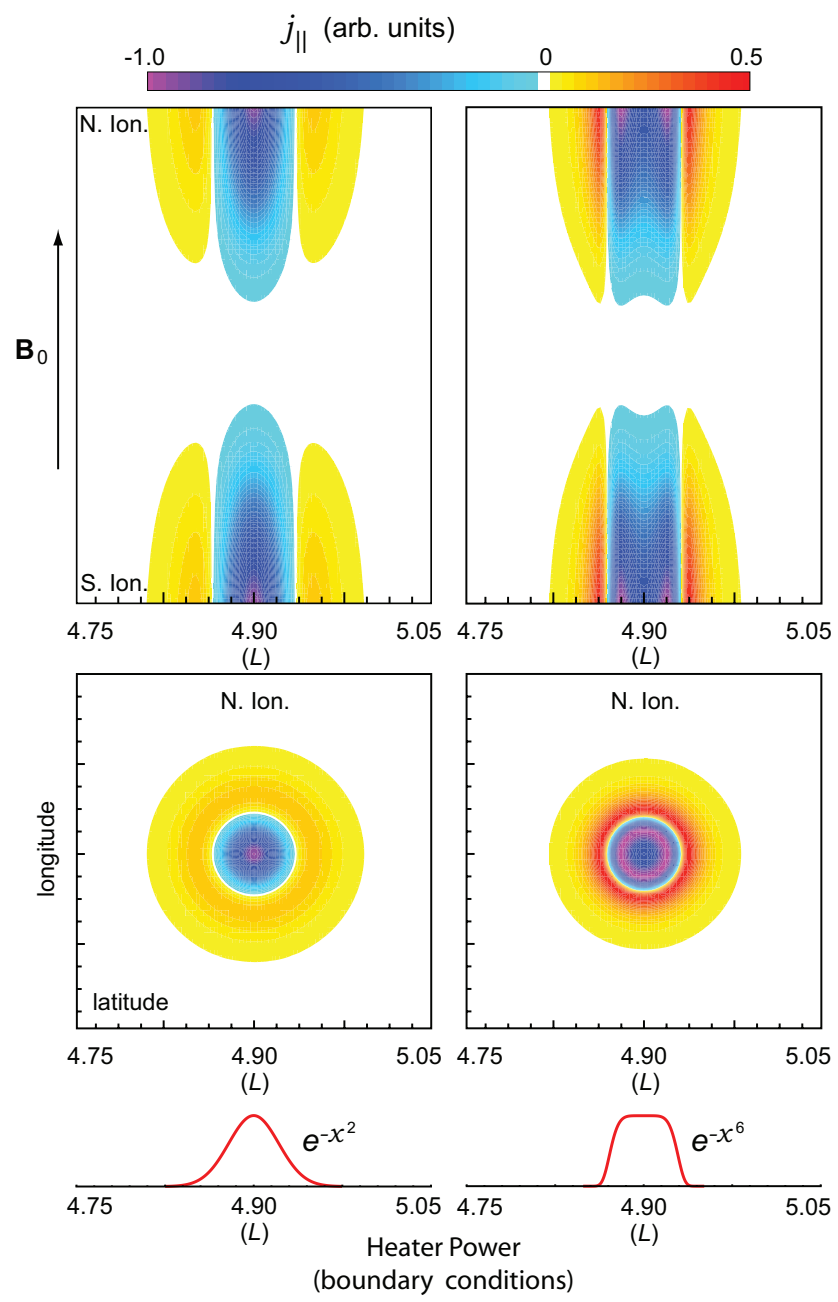

Fig. 4. Left: parallel current density in the magnetosphere (top) and in the ionosphere (middle) from the simulations driven with a zero-frequency driver with $e^{-x^{2}}$ distribution of power across the magnetic field (bottom). Right: the same quantities from the simulation driven with a zero-frequency driver with $e^{-x^{6}}$ distribution of power across the ambient magnetic field.

are (1) strong localization across the ambient magnetic field and (2) distinctive non-symmetrical ionospheric signatures (rings/spots) in the magnetically conjugate locations.

However, there are a number of important differences between these simulations and the spot/ring patterns previously observed, including the one presented for reference in Fig. 1. First, the simulation requires high E-region density/conductivity equivalent to that produced in an active aurora or daylight, whereas the observed rings were produced in the F-region at night when E-region densities are negligible. Second, the optical emissions in F-region experiments originate at altitudes of 150-250 km near specific plasma resonances (Pedersen et al., 2010). Optical emissions driven by FAC, on the other hand, would not be expected to maximize near any particular frequency on the density profile, although 
they could be at E- or F-region altitudes, or both, depending on the energies of the electrons carrying the FAC. Careful examination of optical emission wavelengths, altitudes, and intensities, preferably in both hemispheres and in conjunction with magnetic measurements, would be required to conclusively identify action/contribution from the hypothesized mechanism proposed in this paper. Although the HAARP location maps along the ambient magnetic field to the southern Pacific Ocean, such observations can, in principle, be performed there from ships and/or planes.

Acknowledgements. The High Frequency Active Auroral Research Program (HAARP) is a Department of Defense project operated jointly by the US Air Force and US Navy. The authors wish to thank Dennis Papadopoulos, Evgeny Mishin, and Chia-Lie Chang for fruitful discussions of the ionospheric heating with HAARP. This work is supported in part by the HAARP project under ONR award N00014-08-1-0439 and DARPA contract HR0011-09-C-0099 to Dartmouth College and by ONR MURI award N00014-07-1-0789 to the University of Maryland. Work at AFRL was sponsored by the Air Force Office of Scientific Research.

Topical Editor I. A. Daglis thanks M. Kosch and K. Papadopoulos for their help in evaluating this paper.

\section{References}

Bernhardt, P., Duncan, L., and Tepley, C.: Artificial airglow excited by high-power HF waves, Science, 242, 1022-1027, doi:10.1126/science.242.4881.1022, 1988.

Bernhardt, P., Tepley, C., and Duncan, L.: Airglow enhancements associated with plasma cavities formed during ionospheric heating experiments, J. Geophys. Res., 94, 9071-9092, doi:10.1029/JA094iA07p09071, 1989.

Cohen, M., Inan, U., and Gołkowski, M.: ELF/VLF wave generation via ionospheric HF heating: Experimental comparison of amplitude modulation, beam painting, and geometric modulation, J. Geophys. Res., 115, A02302, doi:10.1029/2009JA014410, 2010.

Cummings, W. D., O'Sullivan, R. J., and Coleman, P. J.: Standing Alfvén waves in the magnetosphere, J. Geophys. Res., 74, 778793, 1969.

Papadopoulos, K., Chang, C., Vitello, P., and Drobot, A.: On the efficiency of ionospheric ELF generation, Radio Sci., 25, 13111320, 1990.

Papadopoulos, K., Gumerov, N., Shao, X., Doxas, I., and Chang, C.-L.: HF-driven currents in the polar ionosphere, Geophys. Res. Lett., in press, 2011.
Pedersen, T., Gustavsson, B., Mishin, E., MacKenzie, E., Carlson, H., Starks, M., and Mills, T.: Optical ring formation and ionization production in high-power HF heating experiments at HAARP, Geophys. Res. Lett., 36, L18107, doi:10.1029/2009GL040047, 2009.

Pedersen, T., Gustavsson, B., Mishin, E., Kendalll, E., Mills, T., Carlson, H., and Snyder, A.: Creation of artificial ionospheric layers using high-power HF waves, Geophys. Res. Lett., 37, L02106, doi:10.1029/2009GL041895, 2010.

Robinson, T. R., Strangeway, R., Wright, D. M., Davies, J. A., Horne, R. B., Yeoman, T. K., Stocker, A. J., Lester, M., Rietveld, M. T., Mann, I. R., Carlson, C. W., and McFadden, J. P.: FAST observations of ULF waves injected into the magnetosphere by modulated RF heating of the auroral electrojet, Geophys. Res. Lett., 27, 3165-3168, 2000.

Ruohoniemi, J. M., Greenwald, R. A., Baker, K. B., and Samson, J. C.: HF radar observations of Pc 5 field line resonances in the midnight/early morning MLT sector, J. Geophys. Res., 96, 15697-15710, 1991.

Samson, J. C., Wallis, D. D., Hughes, T. J., Greutzberg, F., Ruohoniemi, J. M., and Greenwald, R. A.: Substorm intensifications and field line resonances in the nightside magnetosphere, J. Geophys. Res., 97, 8495-8518, 1992.

Southwood, D. J.: Some features of field line resonances in the magnetosphere, Planet. Space. Sci, 22, 483-491, 1974.

Stasiewicz, K., Bellan, P., Chaston, C., Kletzing, C., Lysak, R., Maggs, J., Pokhotelov, O., Seyler, C., Shukla, P., Stenflo, L., Streltsov, A., and Wahlund, J.-E.: Small scale Alfvénic structure in the aurora, Space. Sci. Rev., 92, 423-533, 2000.

Streltsov, A. and Pedersen, T.: An alternative method for generation of ULF waves by ionospheric heating, Geophys. Res. Lett., 37, L14101, doi:10.1029/2010GL043543, 2010.

Streltsov, A. V.: Dispersive width of the Alfvénic field line resonance, J. Geophys. Res., 104, 22657-22666, 1999.

Streltsov, A. V., Lotko, W., Johnson, J. R., and Cheng, C. Z.: Smallscale, dispersive field line resonances in the hot magnetospheric plasma, J. Geophys. Res., 103, 26559-26572, 1998.

Streltsov, A., Gołkowski, M., Inan, U., and Papadopoulos, K.: Propagation of whistler-mode waves with a modulated frequency in the magnetosphere, J. Geophys. Res., 115, A09209, doi:10.1029/2009JA015155, 2010.

Xu, B.-L., Samson, J. C., Liu, W. W., Creutzberg, F., and Hughes, T. J.: Observations of optical aurora modulated by resonant Alfvén waves, J. Geophys. Res., 98, 11531-11541, 1993.

Yampolski, Y. M., Beley, V. S., Kascheev, S. B., Koloskov, A. V., Somov, V. G., Hysell, D. L., Isham, B., and Kelley, M. C.: Bistatic HF radar diagnostics induced field-aligned irregularities, J. Geophys. Res., 102, 7461-7467, 1997. 